\title{
Organic Food and Human Health: An Instrumental Variables Approach Heinz Welsch*
}

Department of Economics, University of Oldenburg, 26111 Oldenburg, Germany

*Corresponding author: Heinz Welsch, Department of Economics, University of Oldenburg, 26111 Oldenburg, Germany, Tel: +49 441 7984112 ; E-mail: heinz.welsch@uni-oldenburg.de

Received date: Sep 13, 2017; Accepted date: Sep 22, 2017; Published date: Sep 30, 2017

Copyright: $\odot 2017$ Welsch H. This is an open-access article distributed under the terms of the Creative Commons Attribution License, which permits unrestricted use, distribution, and reproduction in any medium, provided the original author and source are credited.

\begin{abstract}
Organic food markets in developed countries have been rapidly expanding in recent years. Though expected health benefits are a major motive for buying organic food (OF), the health effects of consuming OF are uncertain. This study uses survey data from Germany, 2007, to explore the causal relationship between OF consumption and self-rated health status. While it finds strong and statistically significant relationships between health and indicators of the intensity and duration of consumption, these relationships vanish when OF consumption is instrumented by respondents' assessment of the necessity of renewable energy. Since the instrument satisfies usual validity standards, these findings suggest that the OF-health relationship may be spurious due to common unobserved factors, in particular a health-oriented lifestyle.
\end{abstract}

Keywords: Health; Food; Consumption; Organic agriculture

\section{JEL Classifications:}

I12, D12, Q13

\section{Introduction}

The aim to "ensure healthy lives and promote well-being for all" features prominently among the United Nations' Sustainable Development Goals [1]. An important contributor to this goal is the implementation of sustainable food production systems and agricultural practices, in particular those that reduce the use of synthetic fertilizers and pesticides [2]. The use of inorganic nitrogen and phosphorus fertilizers has led to large amounts of nitrates and phosphates in soils and waters and to eutrophication of these waters [3]. Since organic farming avoids the use of these substances, it has been recognized by local, national and supranational authorities and stakeholders as an appropriate response to both environmental and human health concerns related to agriculture (For instance, with respect to the European Union, see European Commission [4]).

The market for organic food has experienced a rapid expansion in many developed countries over the past decades. In Germany, for instance, sales steadily increased from 2.1 billion Euros in 2000 to 5.9 billion in 2009 (www.statista.com). Along with environmental concern, one of the strongest motives for consumers to buy organic products is expectations concerning their health effects [5-8]. Because they are produced without using synthetic fertilizers and pesticides, it is believed that organic products contain less toxic residues, in addition to having more health-promoting compounds, especially antioxidative metabolites, such as vitamins. (According to a literature review by Worthington [9] these beliefs tend to be justified, but the causal chain between indicators of nutritional value and health outcomes is ambiguous $[10,11]$. More recently, a comprehens.

ive meta-analysis of existing studies did not find strong evidence that organic foods are more nutritious than conventional alternatives, though consumption of organic foods can reduce the risk of pesticide exposure [10]. In spite of these expectations, however, conclusive evidence on the human health impacts of organic food is largely lacking (for systematic literature reviews [11,12]). (A systematic literature search [11] identified 62 potentially relevant studies published in peer-reviewed journals from January 1958 through March 2010, of which 12 met predefined selection criteria).

Studies analyzing the human health effects of organic food can be classified into intervention studies and epidemiological (observational) studies [11]. In human intervention studies, a group of people is voluntarily brought into a situation in which two subgroups consume either organically or conventionally produced food for a certain period of time. The outcome variable is typically the bioavailability of healthrelevant compounds in the consuming organisms. In epidemiological studies, a large group of people is studied using self-reported indicators of organic food consumption as an explanatory variable and selfassessed health status as the outcome variable.

The lack of conclusive evidence on the health impacts of organic food may be seen as the consequence of methodological issues that are characteristic of the respective study types. The advantage of intervention studies is that they permit causal interpretation, as they can control for third factors. However, these studies refer to specific food products (tomatoes, carrots, apples) as parts of an otherwise habitual diet. This begs the problem that effects may be diluted [11]. Moreover, these studies are usually of a relatively short duration (up to a few weeks) and therefore unable to identify possible effects of longterm organic food consumption. Intervention studies typically find no differences in health outcomes between organic and conventional food consumption (Controlled trials over 14 to 28 days involving tomatoes, carrots and Golden Delicious apples, respectively, found no significant differences in relevant blood and plasma parameters between organic and conventional exposures [11]. See section 3.4 below for a more detailed discussion).

Epidemiological studies, on the other hand, refer to less specific measures of organic diet, but relationships found between organic food and health indicators may be spurious due to inadequate control for third factors. A study by Rembialkowska et al. [13], for instance, found 
that consumers of organic food assessed their health status significantly better than consumers of conventional food products, but the study acknowledged that this finding may be due to aspects of consumers' lifestyles (e.g., nutritional patterns, living environments, physical activity) that are correlated with organic food consumption (Similarly, relationships between measures of organic food and health outcomes found by Alfven et al. [14] and Kummeling et al. [15] may be driven by unobserved common factors. See section 3.4 below for a more detailed discussion).

Overall, it appears that intervention studies tend to find no relationships between organic food and human health, though they may exist, whereas epidemiological studies tend towards finding such relationships but fail to check whether they are spurious.

The present study addresses the human health impact of organic food, focusing on the presumed direct benefits of organic food consumption for human health, rather than on the indirect links through the avoidance of soil and water pollution. It contributes to the literature by addressing the spurious correlation problem inherent in epidemiological designs by means of an instrumental variables approach. It uses a unique set of survey data elicited in Germany 2007, to explore the causal relationship between the consumption of organic food and self-rated health status. While strong and statistically significant relationships are found between health and indicators of the intensity and duration of organic food consumption (controlling for age, income and the education level), these relationships vanish when the consumption of organic food is instrumented by the degree to which respondents agree to an assertion concerning the necessity of renewable energy. This instrument satisfies common validity checks (A switch from 'complete disagreement' to 'complete agreement' to the renewable energy assertion leads to an increase in the various indicators of organic food consumption by up to 1.4 standard deviations. In addition, this instrument passes the usual weak instrument tests). The vanishing relationship between organic food and health status in the instrumental variable estimations thus suggests that the relationship found in least squares regressions may be spurious due to common third factors (such as a health-oriented lifestyle).

Though practically all large scale social surveys at national and international levels include items concerning respondents' health, items concerning health-relevant aspects of nutrition are typically lacking in these surveys. An exception is the Health Survey for England, which includes questions on the consumption of fried foods, sweets, and fruit or vegetables. However, no differentiation is provided as to whether these are organic or conventional products. To my knowledge, the database used in the present study is unique in that it covers people's health status and the intensity and duration of organic food consumption jointly with potential control and instrumental variables (The data set that comes closest to the present one is the one used by Rembialkowska et al. [13], but this study restricts itself to comparing the life styles and health status of organic and conventional consumers within a purely descriptive design).

As already mentioned, the instrument used in this study is the degree to which people agree to the assertion that a switch to renewable energy is necessary. The rationale for choosing this variable is the idea that (a) the attitude towards renewable energy is sufficiently correlated with people's preference for organic food while (b) being uncorrelated with unobserved third factors of health like physical activity, smoking, drinking and nutrition patterns other than organic/ conventional. Part (a) of this conjecture is supported by first-stage regressions of indicators of organic food consumption on the renewable energy variable, in which the latter turns out to be highly significant and to have strong explanatory power (In addition to health considerations, environmental concern is another important motive for organic food consumption [6]. Environmental concern may thus be a common denominator of organic food consumption and the attitude towards renewable energy). Part (b) is supported by regressing the health variable on the renewable energy variable and the controls (age, income, education level). In these regressions, the energy variable turns out to be insignificant and to have no explanatory power.

This study differs from existing studies in several ways. First, unlike intervention studies, which typically include a relatively small number of subjects, it involves a cross-section of several hundred persons. Second, unlike the rather specific types of organic vs. conventional food considered in intervention studies (e.g., tomatoes, carrots, apples) this study focuses on food labelled "organic" in general. Third, since the data set includes information not just on the intensity of people's organic food consumption but also on how long (if at all) they have been buying organic food, the study is more likely to capture effects of long-term consumption of organic food, if they exist. Finally, to my knowledge, this is the first epidemiological study which pursues an instrumental variable approach to address the issue of unobserved factors in the relationship between organic food consumption and human health.

The paper is organized as follows. Section 2 describes the methodological framework (data and econometric approach). Section 3 presents and discusses the results. Section 4 concludes.

\section{Methodological Framework}

\section{Empirical background and data}

As mentioned in the introduction, the market for organic food in Germany experienced a rapid expansion in recent years. In comparison with traditional distribution channels (direct sale by producers, specialized shops), conventional food shops (including supermarkets and discount shops) have increased the share in the organic food market to more than one third by the middle of the decade [16]. Their marketing campaigns may have contributed to the increase of the organic food market.

This study is based on a survey on several types of proenvironmental consumption, including organic food, which was conducted from July to September 2007 in the region of Hanover, Germany (The region of Hanover has about 1.1 million inhabitants. By the time of the survey, organic food was offered all over the region by food suppliers of various forms (organic food shops, supermarkets, discount shops). Because the survey was originally designed to capture a sufficient number of owners of solar heating systems and subscribers to renewable electricity, it was conducted in several stages. Initially, 963 owners of solar heating systems were sent an invitation to participate in the survey. Of these, 190 requested the questionnaire, and 139 completed it. Similarly, 520 subscribers to green electricity were sent an invitation to participate; 150 requested and 122 completed the questionnaire. In addition 233 face-to-face interviews with randomly sampled persons were conducted, using the same questionnaire. Overall, we have 494 valid questionnaires. In the econometric analysis we account for the stratified nature of our sample by weighting the data appropriately (The share of users of solar thermal systems is $2.5 \%$ and the share of subscribers to green electricity is $12 \%$ [17]. 
The survey includes questions on people's pro-environmental behaviors along with their personal characteristics, including their selfassessed health status, and a number of attitude questions. The question concerning organic food (OF) consumption reads as follows: "When shopping food, I buy products labeled as organic ... 'always', 'regularly', 'occasionally', and 'never". Responses were coded 'always' $=4$, 'regularly' $=3$, 'occasionally' $=2$, 'never' $=1$ and this coding was used to define the variable OFIntensity to be employed in the econometric analysis. Alternatively, dummy variables for the four categories were created, labeled OFAlways etc. In addition, people responding 'always' or 'regularly' were asked "For how long have you been buying a significant portion of food labeled as organic?" with response options 'less than one year, 'between one and two years', 'between two and five years', 'between five and ten years', 'more than ten years. For the main analysis, the latter four categories were merged and a dummy variable OFTime was created which takes values $0=$ 'less than one year', $1=$ 'more than one year' (In addition to the main analysis, experiments with dummy variables for the various categories were carried out, see below). In other words, the dummy variable OFTime captures individuals who have been buying organic food at least 'regularly' for at least one year.

The question on the health status reads as follows: "Overall, how would you rate your general health status?", with response options 'very good', 'good', 'average,' 'poor', 'very poor'. The variable Health was created by coding the responses 'very good' $=5, \ldots$ 'very poor' $=1$.

The last main variable in the econometric analysis (the instrument for organic food consumption) is built from the degree of agreement/ disagreement to/with the assertion "We need a consistent change towards renewable energy", coded 'agree completely' $=4$, 'agree' $=3$, 'disagree' $=2$, 'disagree completely' $=1$. This variable is denoted as Ren Needed.

Additional variables included in the empirical analysis are Age, monthly household income (Income), and a seven-point indicator of the education level (Education).

Table 1 in the Appendix reports the descriptive statistics. As seen, the mean intensity of buying organic food is 2.6 on the four-point scale. About 9\%of the respondents fall into the OFAlways category, but about 58\%have been buying organic food at least 'regularly' for at least one year (OFTime). Table 2 present the correlations of the main variables. Health is correlated with OFIntensity at $r=0.18$ and with OFAlways and OFTime at $r=0.13$. RenNeeded is correlated with OFIntensity, OFAlways and OFTime at $\mathrm{r}=0.34, \mathrm{r}=0.15$ and $\mathrm{r}=0.16$, respectively and correlated with Health at 0.09 .

\section{Methodological background}

A fundamental threat to unbiased/consistent estimation is endogeneity, that is, correlation between explanatory variables and the error term. Such correlation may arise from three sources: omitted variables, reverse causality, and measurement error. In any of these cases, least-squares estimates will be biased or inconsistent [18].

Of particular interest in the present case is omitted variable bias (OVB), arising from correlation between explanatory variables included in the analysis and other explanatory variables that are omitted. Omitted variables may imply that estimated relationships are spurious, that is, they may appear to be significant when in fact they are not. To avoid OVB, estimations should control for all relevant observed variables, but that will not be sufficient if some relevant variables are unobserved, such as health-oriented lifestyles in the present case.

The standard method to deal with endogeneity and spurious regressions is instrumental variables (IV) regression. A valid instrument is a variable that is correlated with the explanatory variables of interest, but does not affect the dependent variable in other ways than through the correlation with the explanatory variables, e.g. through correlation with unobserved factors. IV estimation proceeds by regressing the explanatory variable of interest on the instrument (first stage) and replacing the predicted values of the explanatory variable for its actual values (second stage) (IV procedures are implemented in any common econometric packages). If the conditions for a valid instrument are satisfied, IV estimation yields unbiased/ consistent estimates [18].

\section{The Model and empirical approach}

The main specification is of the following form:

$$
\text { Health }_{i}=\alpha+\beta \text { Food }_{i}+\gamma \text { Controls }_{i}+\varepsilon_{i}
$$

where Healthi is the health status of individual $i$ and Foodi stands for the three indicators OFIntensity, OFAlways and OFTime introduced in the preceding subsection.

Candidates for appropriate control variables (Controlsi) are age, sex, income and education (It is well established that there is a positive link between income and health, as higher income may go along with less unhealthy jobs and may allow people to live in healthier places and to buy more expensive medical treatment [19]. The relationship between education and health is more ambiguous [20], but I include education in order to err on the right side, if at all). In preliminary checks I regressed health on a quadratic polynomial of age and on sex, income and the level of education. I found age-squared and (somewhat surprisingly) sex to be insignificant at any reasonable level of significance. The set of controls thus comprises age, income and the education level.

The instrument used in this study is the degree to which people agree to the assertion that a consistent change towards renewable energy is needed. Choosing this variable as an instrument is based on the idea that the attitude towards renewable energy is sufficiently correlated with people's preference for organic food while being uncorrelated with unobserved health-relevant factors like physical activity, smoking, drinking and nutrition patterns other than organic versus conventional food. The first part of this conjecture reflects the circumstance that environmental concern is another important motive for organic food consumption, in addition to health considerations [6]. Environmental concern may thus be a common denominator of organic food consumption and the attitude towards renewable energy.

The dependent variable in eq. (1) is a five-step ordinal variable. In spite of this, the primary estimation methods will be least squares and two-stage least squares. Complementary results from ordered probit estimations will be presented which suggest that the dependent variable can be treated as cardinal without an appreciable influence on the main results. 
Citation: Welsch H (2017) Organic Food and Human Health: An Instrumental Variables Approach. Health Econ Outcome Res 3: 141. doi:

Page 4 of 7

\section{Results and Discussion}

\section{Correlational analysis}

Columns (1) - (4) in Table 1 present results of OLS Health regressions whereas columns (5) - (8) present the ordered probit counterparts. Regression (1) is a benchmark regression without any indicators of organic food consumption. The controls Age, Income and Education have the expected signs and are (at least weakly) significant. They explain 12.6 of the variance in Health.
Regression (2) adds OFIntensity to the explanatory variables. This raises the explanatory power to $14.3 \%$. The coefficients of the controls are not much affected by the inclusion OFIntensity; only the coefficient of Education drops somewhat in magnitude and becomes insignificant. The coefficient on OFIntensity is positive and weakly significant ( $\mathrm{p}=6.9$ $\%)$. The coefficient size suggests that a change from 'never' to 'always' buying organic food is associated with an increase by $0.489\left(3^{\star} 0.163\right)$ of Health on the five-point scale, or about $60 \%$ of one S.D.

\begin{tabular}{|c|c|c|c|c|c|c|c|c|}
\hline & -1 & -2 & -3 & -4 & -5 & -6 & -7 & -8 \\
\hline \multirow[t]{2}{*}{ Constant } & 3.666 *** & $3.307^{\star \star \star *}$ & $3.620^{* \star *}$ & $3.644^{\star \star *}$ & & & & \\
\hline & -0.269 & -0.336 & -0.256 & -0.268 & & & & \\
\hline \multirow[t]{2}{*}{ Age } & $-0.011^{\star \star \star}$ & $-0.010^{* *}$ & $-0.011^{\star \star \star}$ & $-0.011^{* *}$ & $-0.016^{\star * \star}$ & $-0.017^{\star * \star}$ & $-0.170^{\star * *}$ & $-0.017^{\star \star \star}$ \\
\hline & -0.004 & -0.004 & -0.004 & -0.004 & -0.004 & -0.004 & -0.004 & -0.004 \\
\hline \multirow[t]{2}{*}{ Income } & $0.139^{\star \star \star}$ & $0.135^{\text {***}}$ & $0.140^{\star \star \star}$ & $0.136^{\star * *}$ & $0.096^{* * *}$ & $0.091^{\star *}$ & $0.100^{* * *}$ & $0.088^{* \star}$ \\
\hline & -0.035 & -0.035 & -0.034 & -0.036 & -0.036 & -0.037 & -0.037 & -0.037 \\
\hline \multirow[t]{2}{*}{ Education } & $0.063^{*}$ & 0.052 & $0.063^{\star \star}$ & 0.053 & $0.108^{\star * \star}$ & $0.086^{\star \star}$ & $0.101^{\star \star *}$ & $0.088^{\star \star}$ \\
\hline & -0.034 & -0.034 & -0.032 & -0.034 & -0.036 & -0.036 & -0.036 & -0.037 \\
\hline \multirow[t]{2}{*}{ OFIntensity } & & $0.163^{*}$ & & & & $0.217^{\star \star \star}$ & & \\
\hline & & -0.089 & & & & -0.081 & & \\
\hline \multirow[t]{2}{*}{ OFAlways } & & & $0.952^{\star \star \star}$ & & & & $0.476^{* *}$ & \\
\hline & & & -0.209 & & & & -0.202 & \\
\hline \multirow[t]{2}{*}{ OFTime } & & & & 0.194 & & & & $0.271^{* *}$ \\
\hline & & & & -0.121 & & & & -0.111 \\
\hline \multirow[t]{2}{*}{ Cut Point 1} & & & & & $-1.720^{\star * *}$ & $-1.322^{\star \star *}$ & $-1.735^{\star \star \star}$ & $-1.739^{\star \star \star *}$ \\
\hline & & & & & -0.304 & -0.331 & -0.303 & -0.304 \\
\hline \multirow[t]{2}{*}{ Cut Point 2} & & & & & $-0.531^{*}$ & -0.135 & $-0.551^{*}$ & $-0.558^{*}$ \\
\hline & & & & & -0.295 & -0.33 & -0.294 & -0.294 \\
\hline \multirow[t]{2}{*}{ Cut Point 3} & & & & & $1.011^{\star \star *}$ & $1.429^{\star \star \star}$ & $1.010^{* \star *}$ & $1.001^{* \star *}$ \\
\hline & & & & & -0.3 & -0.339 & -0.298 & -0.298 \\
\hline Observations & 416 & 415 & 415 & 415 & 416 & 415 & 415 & 415 \\
\hline $\mathrm{R}^{2} / \mathrm{Psseudo} \mathrm{R}^{2}$ & 0.126 & 0.143 & 0.163 & 0.135 & 0.035 & 0.043 & 0.041 & 0.039 \\
\hline
\end{tabular}

Table 1: Least-squares and ordered-probit results. dependent variable: health.

Regression (3) includes OFAlways instead of OFIntensity. The R2 is now $16.3 \%$. The coefficient on OFAlways is positive and highly significant. Buying organic food 'always' (instead of never, occasionally or regularly) is associated with an increase in Health by 0.952 or 1.2 S.D (I experimented with including, in addition, dummies for buying organic food 'occasionally' and 'regularly' and found them insignificant. This means that it is mainly the difference between buying organic food 'always' and the other degrees OFIntensity that drives the relationship with the health status).

As seen in regression (4), including a dummy for at least regularly buying organic food for at least one year (OFTime) yields a positive but insignificant coefficient for this variable. The explanatory power of this regression is less than in the preceding two regressions. 
Page 5 of 7

Columns (5) - (8) report the ordered probit counterparts to (1) - (4). The signs and the significance of coefficients are as in the corresponding least squares regressions. Interestingly, the significance of OFIntensity and OFTime is now greater than in the previous regressions. With regard to coefficient sizes, the ratios of the various coefficients are similar as under least squares. In addition, the distances between cut points 1 and 2 and between cut points 2 and 3 are rather similar. All of this suggests that the five-point ordinal variable Health can be treated as a cardinal variable without affecting qualitative results.

These qualitative results are that there exists a strong positive relationship between self-assessed health status and the intensity of buying organic food. This relationship is particularly strong and highly significant at the top of the intensity scale, that is, for the dummy variable OFAl ways.

It is obvious that these correlations might be spurious. Especially, they could be due to unobserved common factors of health and organic food consumption, e.g. a health-oriented lifestyle with organic food consumption being just one of several elements. This issue will now be addressed by using an instrumental variable for organic food consumption which is supposed to be uncorrelated to those third factors.

\section{Assessing the instrumental variable}

Columns (1) - (3) in Table 2 present first-stage regressions of the indicators of organic food consumption (OFIntensity, OFAlways, OFTime, respectively) on the prospective instrument, RenNeeded, and the controls. RenNeeded is significant in all three regressions and its inclusion is the main driver of explanatory power (R2 and ). In regression (1), a switch in the 4-point RenNeeded variable from 'completely disagree' to 'completely agree' is associated with an increase in OFIntensity by $3^{\star} 0.362=1.09$ or 1.4 standard deviations (S.D.) Strong relationships also exist between RenNeeded and OFAlways and OFTime $\left(3^{\star} 0.04=0.12\right.$ or 0.4 S.D., and $3^{\star} 0.210=0.69$ or 1.3 S.D, respectively), see regressions (2) and (3). Additionally, the values of the F-statistics generally confirm the absence of weak instrument problems.

\begin{tabular}{|c|c|c|c|c|c|c|c|}
\hline & -1 & -2 & -3 & -4 & -5 & -6 & -7 \\
\hline & OFIntensity & OFAlways & OFTime & Health & Health & Health & Health \\
\hline \multirow[t]{2}{*}{ RenNeeded } & $0.362^{\star \star \star}$ & $0.040^{* *}$ & $0.210^{\star * *}$ & 0.027 & -0.03 & -0.008 & -0.01 \\
\hline & -0.075 & -0.016 & -0.047 & -0.074 & -0.081 & -0.074 & -0.08 \\
\hline Controls & yes & yes & yes & yes & yes & yes & yes \\
\hline \multirow[t]{2}{*}{ OFIntensity } & & & & & $0.170^{*}$ & & \\
\hline & & & & & -0.097 & & \\
\hline \multirow[t]{2}{*}{ OFAlways } & & & & & & $0.951^{\star * *}$ & \\
\hline & & & & & & -0.215 & \\
\hline \multirow[t]{2}{*}{ OFTime } & & & & & & & 0.192 \\
\hline & & & & & & & -0.131 \\
\hline Observations & 416 & 416 & 416 & 415 & 414 & 414 & 414 \\
\hline F-statistic & 20.196 & 3.389 & 13.866 & 14.793 & 13.48 & 15.82 & 12.525 \\
\hline Prob (F-stat.) & 0 & 0.0096 & 0 & 0 & 0 & 0 & 0 \\
\hline $\mathbf{R}^{2}$ & 0.164 & 0.032 & 0.119 & 0.126 & 0.142 & 0.162 & 0.133 \\
\hline$\Delta \mathbf{R}^{2}$ & 0.128 & 0.029 & 0.099 & 0 & -0.001 & -0.001 & -0.002 \\
\hline
\end{tabular}

Notes. Least-squares estimates. Heteroskedasticity-consistent standard errors in parentheses. Controls are Age, Income, Education and a Constant. ${ }^{*} \mathrm{p}<0.10$, ${ }^{* *} p<0.05,{ }^{* * *} p<0.01 . \Delta R^{2}$ is the increase in $R^{2}$ due to inclusion of RenNeeded.

Table 2: Assessment of instrument.

A possible exception is the case of OFAlways, where the effect size and the F-statistic are smaller than in the other two cases. The relative weakness of the instrument in this case is likely due to the large standard deviation of the dependent variable (which is more than three times the mean, see Table 1). However, as argued by Angrist and Pischke [18], weak instruments do not need to be a major problem in just identified models like the one used here.
Columns (4)-(7) in Table 2 check whether there is a relationship between Health and RenNeeded. These regressions are counterparts to the Health regressions (1)-(4) in Table 1, which are here augmented by including RenNeeded. As seen, inclusion of RenNeeded leaves the respective original Health regressions virtually unaffected. In addition, RenNeeded is entirely insignificant in regressions (4)-(7) in Table 2. Any relationship between Health and RenNeeded thus rests on the latter variable's correlation with the organic food variables demonstrated in columns (1)-(3). 
Page 6 of 7

\section{Instrumental variable results}

Table 3 reports the instrumental variable regressions of Health on the various organic food variables. Columns (1)-(3) are the counterparts to columns (2)-(4) in Table 1. Comparing the two tables, it can be noted that the explanatory power of the IV regressions is practically the same as in the corresponding least squares regressions and that the results for the control variables (sign, magnitude and significance of coefficients) are also virtually unchanged.

\begin{tabular}{|c|c|c|c|}
\hline & -1 & -2 & -3 \\
\hline \multirow[t]{2}{*}{ Constant } & $3.526^{\star \star *}$ & $3.672^{* * *}$ & $3.693^{* * *}$ \\
\hline & -0.534 & -0.278 & -0.273 \\
\hline \multirow[t]{2}{*}{ Age } & $-0.011^{* *}$ & $-0.011^{* * *}$ & $-0.011^{* * *}$ \\
\hline & -0.004 & -0.004 & -0.004 \\
\hline \multirow[t]{2}{*}{ Income } & $0.135^{\star \star \star}$ & $0.139^{\star \star \star}$ & $0.136^{* \star *}$ \\
\hline & -0.035 & -0.034 & $(0.036)$ \\
\hline \multirow[t]{2}{*}{ Education } & 0.053 & $0.060^{* *}$ & 0.053 \\
\hline & -0.036 & -0.033 & -0.037 \\
\hline \multirow[t]{2}{*}{ OFIntensity } & 0.084 & & \\
\hline & -0.209 & & \\
\hline \multirow[t]{2}{*}{ OFAlways } & & 0.739 & \\
\hline & & -1.816 & \\
\hline \multirow[t]{2}{*}{ OFTime } & & & 0.142 \\
\hline & & & -0.354 \\
\hline Observations & 414 & 414 & 414 \\
\hline $\mathbf{R}^{2}$ & 0.136 & 0.16 & 0.132 \\
\hline
\end{tabular}

Notes. OFIntensity, OFAlways and OfTime are instrumented by Ren Needed. Heteroskedasticity-consistent standard errors in parentheses. ${ }^{*} p<0.10$, ${ }^{* *} p<0.05,{ }^{* * *} p<0.01$

Table 3: Instrumental-variables results. dependent variable: health.

This is different with respect to the organic food variables. In the first place, the magnitude of the respective coefficients drops. In particular, the coefficient of OFIntensity drops by almost one half. In addition, the coefficients of the organic food variables become insignificant, with $\mathrm{t}$-statistics dropping to less than 0.41 and the corresponding $\mathrm{p}$-values rising to more than 0.68 .

Though low precision of point estimates is common in IV regressions [18], the IV results certainly do not provide much support for a causal relationship between the intensity and duration of organic food consumption and health. Rather, provided that the instrument is valid, they suggest that with probability greater than $68 \%$ (p-values) the least squares relationships are spurious.

An obvious explanation for this could be that the strong and significant relationships between organic food consumption and health found in least squares regressions reflect common unobserved factors related to both variables, in particular health-oriented behaviors correlated with, yet different from organic food consumption.
According to the results presented here, any health effects of organic food are indistinguishable from the effects of those behaviors.

\section{Discussion}

According to Huber et al. [11], there are only a few epidemiological studies investigating the health effects on humans of organic compared with conventional foods. Alfven et al. [14] found that children representing an anthroposophical lifestyle (including biodynamic and organic food) had fewer allergies. Kummeling et al. [15] found an association of strictly organic dairy products with a reduced risk of eczema in infants. Finally, Rembialkowska et al. [13] found that consumers of organic food had a significantly better self-assessed health status than consumers of conventional food.

An obvious problem with these previous epidemiological studies is the possibility that the measures of organic food consumption considered in these studies may be correlated with health-relevant aspects of consumers' lifestyles like physical activity, living conditions and nutritional patterns. Indeed, Rembialkowska et al. [13] found significant differences in these parameters between organic and conventional consumers (That study limits itself to a set of bivariate relationships between organic/conventional food consumption on the one hand and various characteristics and behaviors of the respondents on the other. No attempt at multivariate or even causal analysis is made).

In contrast to epidemiological studies, intervention studies (controlled trials) typically found no significant difference in health outcomes between organic and conventional exposures [21-23]. However, these studies refer to specific food products (tomatoes, carrots, Golden Delicious apples, respectively) as parts of an otherwise habitual diet. This begs the problem that effects may have been diluted [11]. In addition, the duration of controlled trials (14-28 days) may be too short to identify any long-term effects, should they exist.

The present study has focused on food products labeled as organic in general, rather than on specific products. As the cross-sectional data include information on how long people have been buying a sizeable quantity of organic food (if at all), it is in principle possible to detect effects of long-term consumption. In contrast to other epidemiological studies, the present study has addressed the problem of unobserved common factors, especially lifestyles, by means of an instrumental variables approach. Similar as in other epidemiological studies, a significant correlation between health status and organic food consumption was found, but the instrumental variables estimates suggest that these correlations may be spurious.

The instrument used in this exercise was the degree of agreement to the assertion that a consistent switch to renewable energies is needed. This instrument is strongly correlated with organic food consumption because, in addition to health considerations, a strong motive for buying organic food is environmental concern. On the other hand, the instrument is unlikely to be strongly related to behaviors like (non-)smoking, (non-)drinking or (a lack of) physical activity that are characteristic of a health-oriented lifestyle along with organic food consumption.

\section{Conclusion}

Though expected health benefits are an important determinant of the rapidly expanding consumption of organic food, reliable evidence on the causal relationship between organic food consumption and 
human health is surprisingly scarce, and results of existing studies are mixed. While human intervention studies typically do not find significant differences in health outcomes between exposures to specific organic vs. conventional food products, some epidemiological studies have yielded significant positive relationships between organic food and indicators of health status. However, the available epidemiological studies either did not focus specifically on organic food (but on more encompassing styles of nutrition) or failed to control for unobserved common factors of health and organic food consumption.

Using a set of survey data from Germany, this study has applied an instrumental variables approach to investigate the causal relationship between indicators of the intensity and duration of buying organic food and self-reported health status. While least-squares regressions yielded a significant positive relationship between the intensity of organic food consumption and self-assessed health, this relationship vanished when the organic food variables were instrumented by the degree of agreement to an assertion on the necessity of a consistent change towards renewable energies. One explanation for this finding is that consumers of organic food may have a healthier lifestyle overall which, being unobserved, leads to a positive but spurious relationship between organic food and health status in least squares regressions.

While this paper seems to be the first to apply instrumental variables techniques to investigating the causal relationship between organic food and human health, limitations to this study are obvious. The first is possible measurement error, given that all variables used are self-reports. In particular, people may have different ideas about what constitutes a 'good' health status. However, this is a common issue when using subjective data and will not lead to biased results unless measurement error is systematic (non-random). Second, though the instrument used seems to have sufficient power, it is not exogenous. In this regard, a natural experiment design would clearly be desirable, but does not seem to be feasible given available data. Finally, the data used refer to a particular region in Germany. It is not clear if and to what extent they can be generalized to other regions within or outside Germany.

With respect to policy implications, we note that our results should not be construed as implying a refusal of organic farming and its support by government and non-government organizations, as this paper focused entirely on the direct health consequences of organic food consumption. Other, more indirect benefits that stem from the avoidance of fertilizer and pesticide related environmental degradation constitute public goods that definitely warrant fostering food production by organic practices.

\section{References}

1. United Nations (2015) Sustainable Development Goals.

2. Asumadu S, Owusu PA (2016) The Causal Nexus between Child Mortality Rate, Fertility Rate, GDP, Household Final Consumption Expenditure and Food Production Index. Cogent Econom Finance 4: 1191985.

3. Billen G, Garnier J, Lassaletta L (2013) The Nitrogen Cascade from Agricultural Soils to the Sea: Modelling Nitrogen Transfers at Regional Watershed and Global Scales. Philosophical Transactions of the Royal Society of London B: Biologic Sci 368: 20130123.
4. European Commission (2017) Agriculture and the Environment.

5. Harper GC, Makatouni A (2002) Consumer perception of organic food production and farm animal welfare0. British Food J 104: 287-299.

6. Magnusson MK, Arvola A, Hursti UK, Aberg L, Sjoden PO (2003) Choice of organic food is related to perceived consequences for human health and to environmentally friendly behavior. Appetite 40: 109-117.

7. Yiridoe EK, Bonti-Ankomah S, Martin RC (2005) Comparison of Consumer Perceptions and Preference Toward Organic versus Conventionally Produced Foods: A Review and Update of the Literature. Renew Agr Food Syst 2: 193-205.

8. Wier M, Jensen KO, Andersen LM, Millock K (2008) The Character of Demand in Mature Organic Food Markets: Great Britain and Denmark Compared. Food Policy 33: 406-421.

9. Worthington V (2001) Nutritional quality of organic versus conventional fruits, vegetables, and grains, J Altern Comple Med 7: 161-173.

10. Smith-Spangler C, Brandeau ML, Hunter GE, Bavinger JC, Pearson M, et al. (2012) Are Organic Foods Safer or Healthier Than Conventional Alternatives? A Systematic Review. Annals Intern Med 157: 348-366.

11. Dangour AD, Lock K, Hayter A, Aikenhead A, Allen E, et al. (2010) Nutrition-related health effects of organic foods: a systematic review. American J Clin Med 92: 2003-2010.

12. Huber M, Rembialkowska E, Srednicka D, Bügel S, van de Vijver LPL (2010) Organic food and impact on human health: Assessing the status quo and prospects of research. NJAS - Wageningen J Life Sci 58: 101-109.

13. Rembialkowska E, Kazimierczak R, Srednicka D, Bienko K, Bielska M (2008) Different aspects of organic and conventional food consumers' lifestyle. New Med 1: 16-19.

14. Alfven T, Braun-Fahrlander C, Brunekreef B, von Mutius E, Riedler J, et al. (2006) Allergic diseases and atopic sensitization in children related to farming and anthroposophic lifestyle - the PARSIFAL study. Allergy 61: 414-421.

15. Kummeling L, Thijs C, Huber M, van de Vijver LP, Snijders BE, et al. (2008) Consumption of organic foods and risk of atopic disease during the first 2 years of life in the Netherlands. British J Nutri 99: 598-605.

16. Hamm U, Gronefeld F, Halpin D (2004) The European Market for Organic Food: Revised and Updated Analysis. Organic Marketing Initiatives and Rural Development, Vol. 5. University of Wales; Aberystwyth, UK.

17. Clausen J (2008) Feldvermessungsstudie Klimaschutzregion Hannover Borderstep Institut für Innovation und Nachhaltigkeit, Hanover, Germany.

18. Angrist JD, Pischke JS (2009) Mostly Harmless Econometrics-An Empiricist's Companion, Princeton University Press.

19. Goldman N (2001) Social inequalities in health disentangling the underlying mechanisms. Ann N Y Acad Sci 954: 118-139.

20. Braakmann N (2012) The Causal Relationship between Education, Health and Health-Related Behaviour: Evidence from a Natural Experiment in England. J Health Econom 30: 753-763.

21. Caris-Veyrat C, Amiot MJ, Tyssandier V, Grasselly D, Buret M, et al. (2004) Influence of organic versus conventional agricultural practice on the antioxidant micro-constituent content of tomatoes and derived purees: consequences on antioxidant plasma status in humans. J Agri Food Chem 52: 6503-6509.

22. Stracke BA, Rüfer CE, Bub A, Briviba K, Seifert S, et al. (2009) Bioavailability and nutritional effects of carotenoids from organically and conventionally produced carrots in healthy men, British J Nutri 101: 1664-1672.

23. Stracke BA, Rüfer CE, Bub A, Seifert S, Weibel FP, et al. (2010) No effect of the farming system (organic/conventional) on the bioavailability of apple (Malus domestica Bork, cultivar Golden Delicious) polyphenols in healthy men: a comparative study. European J Nutri 5: 301-310. 